\title{
A spectral re-examination of the Markarian AGN
}

\author{
Hartmut Winkler \\ Dept. of Physics, University of Johannesburg, \\ PO Box 524, 2006 Auckland Park, Johannesburg, South Africa \\ email: hwinkler@uj.ac.za
}

\begin{abstract}
The recently published ZORROASTER catalogue is being expanded into what will eventually be a complete list of known 'nearby' $(z<0.1)$ AGN, with optical spectral images, detailed spectral descriptors and waveband-specific flux ratios included where available. The first version of ZORROASTER contained 2843 entries characterised by confirmed or previously suspected broad H-beta emission, and 1000 spectral images. The 2nd version of ZORROASTER was released during the conference, and (in addition to serendipitous updates) includes all $z<0.1$ objects from the list of Markarian. Activity classes and spectral descriptions of Markarian objects have been reviewed, and comparisons of flux ratios derived from the 100 ZORROASTER wavelength-specific pass bands are presented for those Markarian AGN for which Sloan Digital Sky Survey spectra exist. The value of the improved parameterizations and classifications of the Markarian objects on AGN studies, and the implications on some past investigations based on the Markarian lists, are briefly discussed.
\end{abstract}

Keywords. galaxies: active, galaxies: Seyfert, catalogs, astronomical data bases: miscellaneous

\section{Introduction}

The survey of galaxies with ultraviolet continua by Markarian and collaborators at Byurakan Observatory yielded a list of 1515 such objects, published in a series of fifteen papers beginning with Markarian (1967) and completed with Markarian et al. (1981). The list contained many then unknown objects with active galactic nuclei (AGN), which were poorly understood at that time. The Markarian objects (as they became soon known as) thus provided a timely expansion of the sample of known AGN, and studies of various Markarian AGN contributed significantly to the development of theories explaining the AGN phenomenon (e.g. Osterbrock \& Dahari 1983, Osterbrock \& Pogge 1985).

Even though entries on the Markarian list were all identified in a common manner based on their apparent blueness, they constitute a diverse set of astrophysical objects, ranging from nearby blue stars superimposed on normal galaxies to distant quasars (Mazzarella \& Balzano 1986). In almost all cases emission lines have been confirmed in follow-up studies (e.g. Arakelian et al. 1970, Markarian et al. 1980), but accurate spectral classification has often not been possible due to the noisiness of the available spectra. Even in the most recent compilation of the properties of the Markarian list objects (Petrosian et al. 2007), the spectral information remains uncertain for a significant fraction of entries.

Over the decades following the publication of the Markarian lists, there have been numerous attempts to study the collective properties of specific types of AGN using samples that usually included a significant proportion of Markarian objects (e.g. Yee 1983, Meurs \& Wilson 1984, Reichert et al. 2004). Here the spectral uncertainty associated with some objects can effectively contaminate a sample, and affect the properties determined for the AGN class. It is therefore still important that the spectral nature of all objects on the Markarian list be established without a doubt. Even in the case of well observed 


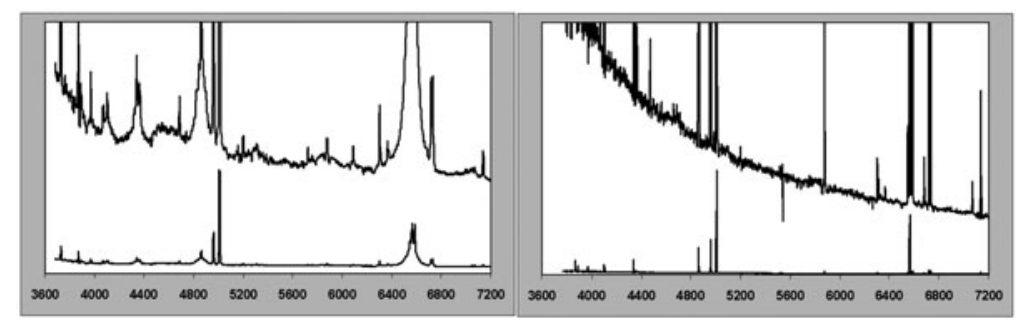

Figure 1. Examples of ZORROASTER spectral images: Mkn 1392 (left), Mkn 1236 (right). Spectra are plotted at two vertical scales, and the horizontal axis displays rest wavelength in $\AA$.

objects, reviewing the spectral type may be desirable, especially when the nuclei are prone to large variations (e.g. Cohen et al. 1986). The importance of this exercise is highlighted by the fact that yet another presentation at the conference addressed the very same topic (Mickaelian et al. 2014).

\section{The spectral re-classification}

The advent of several large-scale programmes involving the systematic collection of the optical spectra of thousands of targets is greatly broadening the database of quality spectra for Markarian objects. The Sloan Digital Sky Survey (SDSS) (Ahn et al. 2013) has proved particularly useful in this regard, especially as it covers a part of the sky in which many Markarian objects are also located. The spectra of 773 objects $(51 \%$ of the Markarian list) are available on SDSS.

Following the tradition of the well known Veron AGN catalogue (Veron-Cetty \& Veron 2010), which is no longer being updated (M.-P. Veron-Cetty, private communication), the author has in recent years built up a catalogue named Z-limited Optical Region Register Of AGN Spectra, Types and Emission Ratios (ZORROASTER). While the initial version of ZORROASTER only included broad-line AGN at $z<0.1$, the updated version released during the conference (Winkler 2013) includes a supplement with the complete Markarian list.

The spectral classification in ZORROASTER differs from previous AGN catalogues in that it attempts to specify optical spectral characteristics in more detail than is normally encountered. Additional descriptors are used to highlight features such as broad line width and profile, iron band strength, helium line emission, coronal line presence, starbursts and host galaxy spectrum. On top of this, common format spectral images are embedded in the catalogue to enable independent 'quick look' classifications. Examples of such spectral images may be viewed in Fig. 1.

Where no SDSS data was available for an object, the literature was searched for the best spectral image, and the same classification system was applied for these. In that way another 381 objects ( $25 \%$ of the Markarian sample) had their spectra identified. Even after this search, however, 361 objects (24\% of Markarian galaxies) did not seem to have published spectra (though in some cases authors had offered classifications), meaning that their nature and activity cannot be readily verified, and must therefore be open to some doubt.

For the 1154 Markarian objects whose spectra could be reviewed, the majority were indeed broadly consistent with the previously catalogued spectral classes. ZORROASTER however does list more detail than previously reported in most cases. There were however also thirty starburst galaxies and starburst-Seyfert hybrids misclassified as conventional Seyferts, nine LINER-Seyfert mixups and several type 1 Seyferts whose broad line 
Table 1. Revised AGN classifications. The old class is from Veron-Cetty \& Veron 2010

\begin{tabular}{|l|l|l|}
\hline Mkn & old class & reclassified to \\
\hline 40 & Sy 1.0 & Seyfert (Sy) 1.5 \\
69 & Sy 1.0 & Sy 1.5 - starburst hybrid \\
590 & Sy 1.0 & Sy 1.8 \\
728 & Sy 1.9 & Sy 1.5 \\
783 & NLS1 & Sy 1.8 with peculiar asymmetric broad lines \\
845 & Sy 1.0 & Sy 1.8 \\
926 & Sy 1.5 & peculiar Sy 1.8 (see discussion below) \\
1018 & Sy 1.9 & Sy 1.5 \\
1102 & Sy 2 & medium excitation starburst galaxy \\
1103 & Sy 2 & medium excitation starburst galaxy \\
1119 & Sy? & strongly reddened Sy 1.2 \\
1179 & Sy 1.9 & Sy 1.2 \\
1310 & Sy 1.0 & Sy 1.5 - starburst hybrid \\
\hline
\end{tabular}

strength was very different to what was previously reported (probably a sign of nuclear variability). A summary of the more interesting reclassifications is presented in Table 1.

\section{Spectral line measurements and classification}

Another feature of the ZORROASTER database is that for many of the entries processed to date, including 754 Markarian objects, a series of synthetic photometric measurements were generated from the SDSS spectra. These record the flux through 100 narrow pass bands coinciding with specific spectral features. They are used to quantify continuum levels as well as specific line strengths, widths and profiles. From these values object characteristics such as ionisation, temperature, density, gas dynamics, extinction, element abundances, spectral peculiarities and host galaxy properties can be parameterised and compared. It is even possible to generate cross-plots of particular line ratios similar to the diagnostic diagrams frequently employed for AGN classification (e.g. Kewley et al. 2006).

The ZORROASTER catalogue and selected band ratios and properties obtained through these can be accessed online (Winkler 2013).

\section{Notes on two peculiar objects}

Mkn 17\%. This object has previously generated little interest. The galaxy was known to have an emission spectrum, and the SDSS data confirm the presence of relatively modest emission lines typical of a medium ionisation star forming region. SDSS however also has a spectrum of the bright spot south-west of the galaxy centre (Fig. 2). What otherwise looks like a foreground star in fact displays strong broad emission lines, at a redshift with a value similar to the rest of the galaxy. The spectrum of the bright spot resembles that of a type 1 Seyfert nucleus in many ways, including the presence of the forbidden lines. A closer look at the broad Balmer lines however highlight their P Cygni profile, making this a likely and previously unreported supernova (the forbidden lines presumably indicating that the bright spot is also in a star forming region).

Mkn 926. This galaxy, also known as MCG -2-58-22, was at the time of its discovery one of the most luminous nearby quasars (Ward et al. 1978). Its brightness has however steadily decreased in recent years, and in the SDSS spectrum the broad Balmer lines are both weak and asymmetric. Furthermore, the comparatively strong [O I] and [S II] lines are reminiscent of spectra of broad-line LINERs. This link between LINER properties and bright quasar phases make this object unique and deserving of further investigation. 


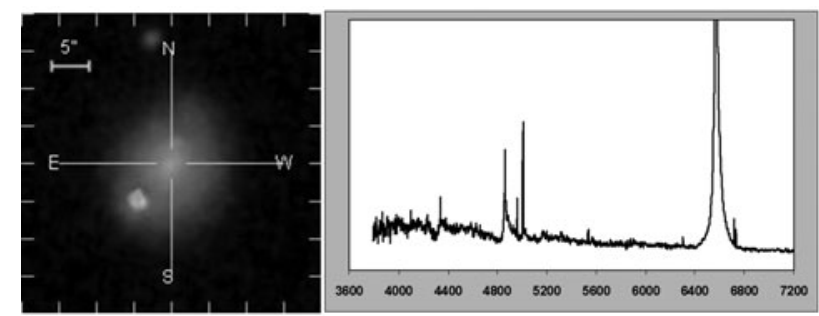

Figure 2. SDSS image of Mkn 177 and SDSS spectrum of the off-centre bright spot.

Acknowledgements. The author dedicates this paper to the late Tony Fairall, who inspired his interest in AGN surveys. Fairall was himself strongly influenced by the work of Markarian, which in turn motivated the southern hemisphere Fairall list of galaxies.

This research has made use of the NASA/IPAC Extragalactic Database (NED) which is operated by the Jet Propulsion Laboratory, California Institute of Technology, under contract with the National Aeronautics and Space Administration.

Funding for SDSS-III has been provided by the Alfred P. Sloan Foundation, the Participating Institutions, the National Science Foundation, and the U.S. Department of Energy Office of Science. The SDSS-III web site is http://www.sdss3.org/.

SDSS-III is managed by the Astrophysical Research Consortium for the Participating Institutions of the SDSS-III Collaboration including the University of Arizona, the Brazilian Participation Group, Brookhaven National Laboratory, Carnegie Mellon University, University of Florida, the French Participation Group, the German Participation Group, Harvard University, the Instituto de Astrofisica de Canarias, the Michigan State/Notre Dame/JINA Participation Group, Johns Hopkins University, Lawrence Berkeley National Laboratory, Max Planck Institute for Astrophysics, Max Planck Institute for Extraterrestrial Physics, New Mexico State University, New York University, Ohio State University, Pennsylvania State University, University of Portsmouth, Princeton University, the Spanish Participation Group, University of Tokyo, University of Utah, Vanderbilt University, University of Virginia, University of Washington, and Yale University.

\section{References}

Ahn, C. P., Alexandroff, R., Allende Prieto, C., Anders, F., et al. 2013, arXiv, 307.7735A

Arakelyan, M. A., Dibai, E. A., \& Esipov, R. C., V. F. 1970, Astrophysics, 6, 14

Cohen, R. D., Rudy, R. J., Puetter, R. C., Ake, T. B., \& Foltz, C. B. 1986, ApJ, 311, 135

Kewley, L. J., Groves, B., Kauffmann, G., \& Heckman, T. 2006, MNRAS, 372, 961

Markarian, B. E. 1967, Astrofizika, 3, 55

Markarian, B. E., Lipotevskii, V. A., \& Stepanian, D. A. 1980, Astrofizika, 16, 5

Markarian, B. E., Lipotevskii, V. A., \& Stepanian, D. A. 1981, Astrofizika, 17, 619

Mazzarella, J. M. \& Balzano, V. A. 1986, ApJS, 62, 751

Meurs, E. J. A.. \& Wilson, A. S. 1984, A\& $A, 136,206$

Mickaelian, A. M., Abrahamyan, H. V., Harutyunyan, G. S., \& Paronyan, G. M. 2014, these proceedings

Osterbrock, D. E. \& Dahari, O. 1983, ApJ, 273, 478

Osterbrock, D. E. \& Pogge, R. W. 1985, ApJ, 297, 166

Petrosian, A., McLean, B., Allen, R. J., \& MacKenty, J. W. 2007, ApJS, 170, 33

Reichert, D. S., Lüdke, E., \& Riffel, R. A. 2004, in: T. Storchi-Bergmann, L. C. Ho \& H. R. Schmitt (eds.), Proceedings of IAU Symposium 222 (Cambridge University Press), p. 357

Veron-Cetty, M.-P. \& Veron, P. 2010, A\&̊A, 518, A10

Ward, M. J., Wilson, A. S., Penston, M. V., Elvis, M., Maccacaro, T., \& Tritton, K. P. 2007, ApJ, 223, 788

Winkler, H. 2013, www.uj.ac.za/EN/Faculties/science/departments/physics/Pages/zorroaster. aspx

Yee, H. K. C.. 2006, ApJ, 272, 473 